\title{
Transradial approach to lower extremity interventions
}

\author{
This article was published in the following Dove Press journal: \\ Vascular Health and Risk Management \\ 19 June 2010 \\ Number of times this article has been viewed
}

\author{
Ravikiran Korabathina' \\ Sidharth S Yadav' \\ John T Coppola ${ }^{2}$ \\ Cezar S Staniloae ${ }^{2}$ \\ 'Department of Cardiovascular \\ Medicine, Saint Vincents Catholic \\ Medical Center, New York, NY, USA; \\ ${ }^{2} \mathrm{Cardiac}$ and Vascular Institute, \\ New York University Medical Center, \\ New York, NY, USA
}

\begin{abstract}
Percutaneous interventions of the coronary and peripheral vessels have historically been performed using a femoral artery approach. There has been increasing recognition of postprocedural bleeding complications and its impact on short- and long-term mortality. Because of its now recognized safety, the transradial approach has recently emerged as a preferred method compared to the transfemoral approach. The limitations associated with the distance from the puncture site to the lesion location are being addressed as new tools are developed for the endovascular treatment of peripheral arterial disease. In this review, we discuss the many facets of the transradial approach to lower extremity endovascular interventions, highlighting its safety and efficacy. Approaches to special populations including individuals with prior surgical bypass, Leriche's syndrome, and those committed to chronic anticoagulation are also reviewed.

Keywords: peripheral arterial disease, endovascular interventions, transradial interventions, aorto-iliac angioplasty
\end{abstract}

\section{Introduction}

Percutaneous interventions of the coronary and peripheral vessels have historically been performed using a femoral artery approach. Alternative access sites, including the brachial and axillary arteries, have been employed in clinical scenarios where groin access is contraindicated. Nevertheless, these approaches have been associated with a relatively high risk of vascular and nerve complications. Furthermore, cannulation of these arteries has the potential for thrombotic occlusion which may put the entire distal limb at risk. Recently, post-procedural bleeding complications with a femoral approach have raised safety concerns, due to their contribution to both short- and long-term mortality. As such, the transradial approach (TRA) has gained significant interest, and a growing body of evidence from the coronary literature has demonstrated this method to be safer than the transfemoral approach (TFA), while still maintaining efficacy. ${ }^{1,2}$ Despite this, the TRA technique has not become mainstream in the United States, as a large report from the National Cardiovascular Data Registry has shown a usage rate for coronary procedures of only $1.32 \%$ between $2004-2007$. $^{3}$

In recent years, there has been a surge of interest in percutaneous peripheral vascular interventions. The poly-vascular nature of atherosclerosis that is frequently present in the individual who manifests with peripheral arterial disease (PAD), makes surgical repair options riskier and often precludes the use of such an aggressive approach. As such, there has been a dramatic development of endovascular tools and techniques. It follows that the use of percutaneous modalities coupled with safer access-site strategies, may offer the higher-risk PAD patient the greatest safety margin. Based upon
Correspondence: Cezar S Staniloae New York University Medical Center, Cardiac and Vascular Institute, 550 First Avenue, New York, NY 10016

Tel + I 9175624659

$\mathrm{Fax}+12127277356$

Email cstaniloae@aol.com 
the extensive safety data for the use of the TRA for coronary angiography and interventions, we have extrapolated this technique to interventions of the aorto-iliac arterial system and have published our safety and efficacy data. ${ }^{4,5}$ In this review, we discuss the many facets of the TRA and highlight the feasibility of this technique for endovascular interventions of the lower extremities.

\section{Anatomy}

Whether for coronary or peripheral interventions, it is mandatory that the radial operator be well-versed with normal radial artery anatomy and its anatomical variants. The radial artery in most people starts at the bifurcation of the brachial artery just below the bend of the elbow. It then passes along the radial side of the forearm to the wrist and terminates by passing between the two heads of the first interosseous dorsalis and into the palm. At this level, it loops across the metacarpal bones towards the ulnar side of the hand, uniting with the deep palmar branch of the ulnar artery. ${ }^{6}$ There is extensive communication between the ulnar and radial branches that maintain blood flow to the hand in the event of radial occlusion.

Knowledge of anatomical variants is essential as it allows for anticipation of problems as they may arise during the course of the procedure and facilitates appropriate measures to overcome difficult anatomy. Two large reviews have demonstrated that anatomical variants, including tortuosities, stenoses, hypoplasias, and radioulnar loops occur in up to $23 \%$ of transradial catheterizations. ${ }^{7,8}$ These abnormalities could lead to significantly reduced puncture and procedural success rates.

It is also important to discern the caliber of the radial artery, as this factor has implications regarding equipment selection. An abnormally small caliber of the radial artery may be due to spasm, hypoplasia, or atherosclerosis. In the latter case, the radial artery is not protected from atherosclerotic disease. Histopathologic studies examining the radial artery in patients with coronary artery disease have shown that up to $7 \%$ have severe plaque burden and approximately $21 \%$ have medial calcification. ${ }^{9,10}$ These diseased arteries are more likely to be prone to spasm.

In our experience, we have encountered more spasm in patients with smaller diameter radial arteries. Given that the long sheaths used during peripheral interventions must traverse the entire length of the upper extremity, particular attention must be devoted to the administration of appropriate spasmolytic cocktails and sedation. This is of even greater importance at the end of the case during sheath removal, since the prolonged exposure of the radial artery to the large-sized sheath, may lead to significant spasm.

Anthropometric data, including arm and torso length, are important considerations in the TRA as there are limitations to equipment use in taller individuals. Currently, the longest available sheath is $110 \mathrm{~cm}$, and the longest shaft length for the available stents is $135 \mathrm{~cm}$. In taller patients, accessing the left radial artery as opposed to the right radial artery eliminates about $10-12 \mathrm{~cm}$ in length due to the proximity of the left subclavian artery to the descending aorta.

It is equally important to identify the presence of certain risk factors that may alter the tortuosity and the course of the radial artery, including the presence of long-standing hypertension and older age. However, aside from making sheath delivery difficult, once positioned, these variations do not cause further problems in carrying out peripheral interventions as they do for coronary procedures.

\section{Technical considerations}

The routine approach to the suprainguinal intervention starts with gaining access from the left radial artery. This has the advantage of traversing a shorter distance to the descending aorta and not crossing the aortic arch and cerebral vessels. The left subclavian artery most often directs the angiographer towards the descending aorta, so we usually begin the procedure with a $125 \mathrm{~cm}$ multipurpose catheter and an angle-tipped hydrophilic coated $0.035^{\prime \prime}$ wire. Occasionally, an internal mammary shape catheter may be required to negotiate the descending aorta due to a type III aortic arch. Regardless of the wire used, it is mandatory that its passage is carefully followed with fluoroscopy to its destination in the lower abdominal aorta. Severe complications can result from "blind" advancement of the wire into tributaries of the thoracic or abdominal aorta. We recommend that in most cases, angiography of the aorto-iliac system begins with a pigtail "power" injection of the lower abdominal aorta (typically 20 milliliters over one second in the antero-posterior projection). This initial scout film may help define any aortic aneurysms, collateral vessels, and the presence of ostial disease of the common iliac arteries. This initial view may be forgone only in the presence of renal insufficiency with the backup of excellent non-invasive imaging. Selective angiography of the entire lower extremity is then performed with the 5 Fr $125 \mathrm{~cm}$ multipurpose catheter.

Current stent and balloon platforms of all major manufacturers can be safely accommodated within 6 Fr diameter sheaths. We most commonly use a 6 Fr $110 \mathrm{~cm}$-long sheath (Cook Medical, Bloomington, IN) that is easily positioned 
at the proximal common iliac artery, or just at the distal aorta in case of ostial iliac disease. From this point on, all interventions are performed in the similar fashion as from the femoral approach.

The current available equipment only allows for iliac and proximal superficial femoral artery (SFA) interventions, due to limitations regarding the shaft length. Nevertheless, a recent study showed the potential for effective SFA balloon angioplasty in a small cohort of patients with in-stent restenosis. ${ }^{11}$ This was possible due to the recent availability of $180 \mathrm{~cm}$ balloon shaft lengths (Medtronic Invatec, Bethlehem, PA). However, the current introducer sheaths are a maximum of $110 \mathrm{~cm}$, and the longest shaft length for stent platforms is still limited to $135 \mathrm{~cm}$ (Abbott Vascular, Santa Clara, CA). To complete interventions throughout the entire SFA, stent shafts of at least $160-170 \mathrm{~cm}$ are required. In spite of the current limitations, the potential benefits of the TRA outlined above should justify development of specific devices designed for this purpose.

\section{Safety}

The fundamental reason for the tremendous interest in the TRA compared to any other arterial access sites derives not only from its favorable anatomical distinctiveness, but also its unrivaled safety profile. Most of this safety data stems from the coronary literature, as repeated studies comparing the TRA to the TFA have demonstrated a significant reduction in bleeding complications, further translating into reduced short and long-term mortality. A review of all transradial coronary interventions from the National Cardiovascular Data Registry performed between 2004-2007 showed significantly lower bleeding risks [odds ratio $0.42(0.31-0.56)$ ], even after multivariable adjustment. ${ }^{3}$ Another recent review of 32,822 patients in British Columbia undergoing cardiac procedures noted that access site bleeding complications necessitating blood transfusions conferred a significantly increased 30-day and 1-year mortality [odds ratio 4.01 (3.08 to 5.22) and 3.58 (2.94 to 4.36), respectively]..$^{12}$ The use of a radial technique halved this rate of blood transfusion.

Until recently, there have been no studies comparing the use of the TRA to other arterial access sites for peripheral angioplasty. In the only study published, at the time of this writing, that compared the TRA and TFA in aorto-iliac interventions, our group demonstrated a comparable safety profile between the two approaches. ${ }^{5}$ At our institution, we collected prospective data on 68 subjects with claudication and critical limb ischemia, who underwent aorto-iliac interventions between June 2007 and June 2009. Twenty-seven patients had 33 lesions treated via the TRA, while 41 patients had 47 lesions treated via the TFA. There were no major access site complications (defined as either a hematocrit drop by at least $10 \%$ or surgery/packed red blood cell transfusion requirement to correct bleeding) in either group. Thirty-day major adverse events, including myocardial infarction, stroke, death, target-vessel revascularization, or amputation, were also not present in either group. Only in the TRA group were no minor access-site complications present, defined as persistent bleeding requiring use of additional compressive devices or the development of local hematoma. The TFA group showed a $7.3 \%$ rate of such complications. These findings are consistent with the growing body of evidence from the coronary literature that the TRA is safer than the TFA.

There has been some concern regarding radiation exposure to both the patient and operator, during transradial interventions, as studies from the coronary literature have shown mixed results. Furthermore, there is a perception of higher radiation exposure via the left compared to the right TRA. In interpreting this data, one must be careful to distinguish total fluoroscopy time from total procedural time, as the latter may prove to be more time-consuming for the TRA in terms of dedicated access time and set-up. One coronary study illustrated that the total procedural time was significantly longer with the TRA compared to the TFA (18.1 vs $15.0 \mathrm{~min}$, $P=0.009)$, whereas the fluoroscopy time was not different $(5.6 \pm 5.9$ vs $4.7 \pm 3.9, P=0.47) .{ }^{13}$ In our peripheral study, there was no difference between the TRA and TFA interventions in terms of total fluoroscopy time (TRA: 30.4 min vs TFA: $26.6 \mathrm{~min}, P=0.60$ ), despite the majority of cases being performed from the left TRA. Another study even showed a significant reduction in fluoroscopy and procedural times using the left TRA for coronary catheterizations. ${ }^{14}$ Therefore, concerns about radiation exposure with the left TRA should not dissuade operators from the radial approach for aortoiliac interventions.

\section{Efficacy}

The advantages to the TRA in aorto-iliac angioplasty and stenting extend even beyond its safety to its efficacy. Other than a description of the use of the TRA for lower extremity interventions, ${ }^{15}$ comparison data to other access sites in terms of efficacy endpoints are limited to only two studies. In a small cohort of 12 subjects with SFA in-stent restenosis, one report demonstrated successful balloon angioplasty in all cases using the TRA and marked improvements in walking distance at follow-up. ${ }^{11}$ The second study, presented by our group, looked at the outcomes from 80 aorto-iliac 
interventions. ${ }^{5}$ Both the TRA and TFA groups showed similar mean baseline Rutherford category (2.9 vs 2.6, respectively) and pre-intervention ankle-brachial index ( 0.64 vs 0.67 , respectively). However, lesion parameters differed, as the TRA group was characterized by a threefold higher presence of total occlusions $(27.3 \%$ vs $8.5 \%$, $P=0.03)$ and a greater baseline diameter stenosis (89.2\% vs $82.3 \%, P=0.003)$. Despite this inequality in lesion types, there was no difference in mean procedural time (TRA: 97.9 min vs TFA: $83.4 \mathrm{~min}, P=0.08$ ) or contrast volume requirement (TRA: $238.7 \mathrm{~mL}$ vs TFA: $213.1 \mathrm{~mL}, P=0.35$ ). Interestingly, during the course of the study, the procedural time became shorter by an average of 25 min within the TRA group (112.3 min to $87.6 \mathrm{~min}, P=0.07$ ), suggesting a positive learning curve. In addition, the primary and secondary procedural success rates were similar between the TRA and TFA groups (87.9\% vs $97.8 \%, P=0.15$ and $93.9 \%$ vs $100 \%, P=0.17$, respectively). Post-intervention hemodynamics, as measured by ankle-brachial index, showed similar degrees of improvement (TRA: 0.64 to 0.77 and TFA: 0.67 to $0.85, P=0.77$ ).

Our experience with TRA in aorto-iliac interventions clearly demonstrated that despite the worse case-mix that was present in the TRA group, this technique can be as successful as the TFA. Furthermore, radial access can be a viable option even for more complex peripheral lesion types, as the prevalence of TASCII C and D lesions in our study approached $40 \%$. There are two additional issues that concern beginner radial operators. The first has to do with the anticipated need for larger balloon and stent sizes requiring bigger delivery sheaths, given the larger vessel diameters of the iliac arteries. The second has to do with the longer distance from the puncture site to the target lesion. As shown in our study, interventions in the TRA cohort were not limited to smaller angioplasty devices; in fact the mean stent diameter deployed for the TRA cohort was $9.30 \mathrm{~mm}$ compared to $9.33 \mathrm{~mm}$ for the TFA subjects. Further, all stent platforms were successfully delivered through 6 Fr sheaths. Finally, the distance to the target lesion was never an indication to abort the TRA and resort to the TFA in order to complete the procedure.

\section{Special populations}

Perhaps endovascular interventions for select anatomical and clinical presentations are better served when approached using the TRA. These particular subgroups include individuals with prior surgical bypass, Leriche's syndrome, and those committed to chronic anticoagulation.

\section{Surgical bypass}

Individuals who have undergone previous surgical revascularization, particularly aorto-bifemoral bypass, pose a unique challenge in that the two bypass conduits usually form an acute angle from their anastamotic take-off

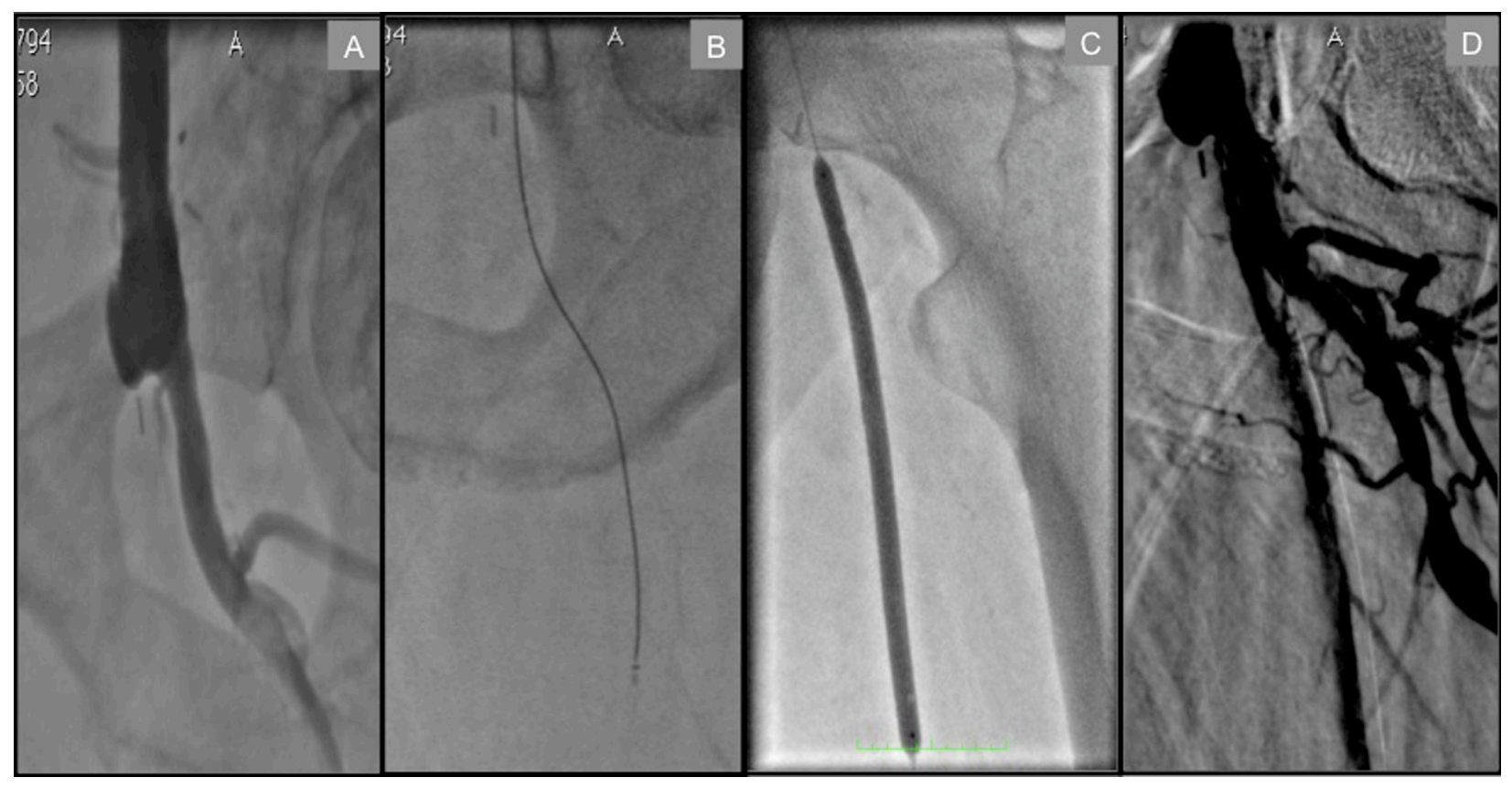

Figure I Transradial approach to a left proximal SFA total occlusion in a patient with a history of aorto-bifemoral bypass. A) Distal anastamosis site of bypass conduit to CFA and total occlusion of SFA at its ostium. B) Crosser CTO recanalization catheter ${ }^{\mathrm{TM}}$ (Flowcardia) successfully passing through proximal total occlusion. C) Balloon angioplasty of proximal SFA. D) Final result at the end of procedure. 
from the distal aorta. The nature of this reconstruction precludes a contralateral retrograde femoral approach to common femoral artery (CFA) or proximal SFA lesions. Meanwhile, the narrow angle formed by the bypass limbs is easily traversed from above via the radial approach, lending even greater support. In Figure 1, we illustrate a proximal SFA chronic total occlusion in a patient with a history of aorto-bifemoral bypass that was successfully treated via the TRA.

\section{Leriche's syndrome}

Atherosclerotic occlusive disease of the infrarenal abdominal aorta and iliac vessels is seldom limited to the suprainguinal territory. In fact, most surgical series have reported that up to two-thirds of individuals presenting with aorto-iliac occlusive disease have infrainguinal disease as well. ${ }^{16}$ Accessing the CFA in such an individual with extensive disease makes them more susceptible to local vascular complications, including dissection, subintimal guidewire passage, and vessel perforation. Furthermore, compressing the CFA after the procedure in the presence of occlusive disease may lead to ischemia or even thrombosis.
In Figure 2, we illustrate a patient who presented with Leriche's syndrome that was successfully recanalized via the TRA.

Certain types of occlusive disease may present relatively acutely requiring urgent attention. The recommended therapies for individuals with acute limb ischemia categorized as Rutherford class I or IIa include catheterdirected thrombolysis. The TRA in such patients would be the safest access strategy as it avoids all of the potential catastrophic complications that could occur from a TFA approach.

\section{Anticoagulation}

Many individuals require chronic anticoagulation with warfarin. A prerequisite to contemplating a lower extremity intervention in such a patient is either bridging therapy with shorter-acting agents or reversal of anti-coagulation with vitamin K or by wash-out. Depending upon the severity of the indication for chronic anticoagulation, this is not an easy task, with its attendant clinical risks during transition and time costs. Given the safety of the TRA in terms of accesssite bleeding, there is essentially no need to discontinue

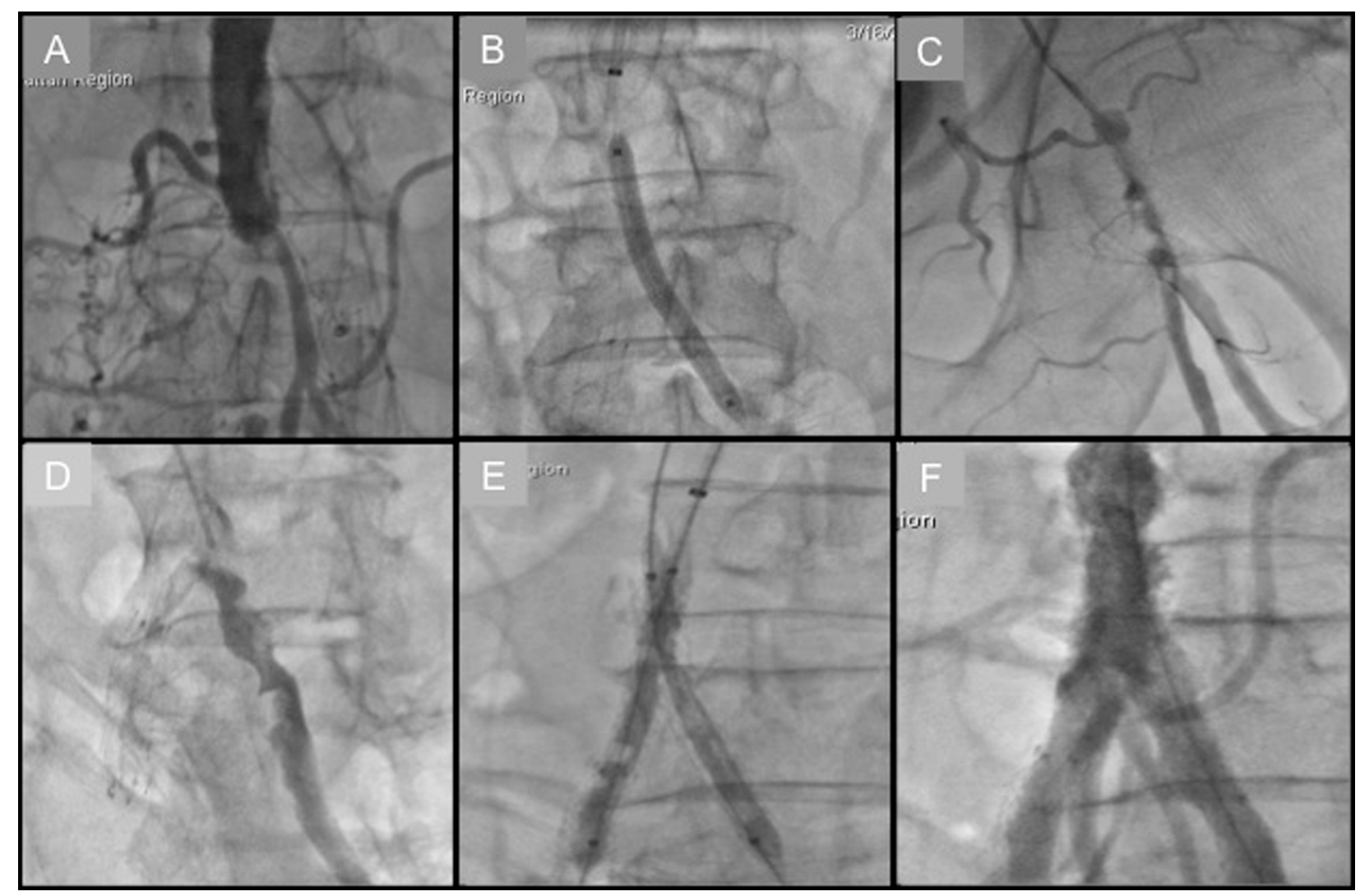

Figure 2 Transradial approach to Leriche's syndrome. A) Aortogram showing distal aorta total occlusion. B) Balloon angioplasty of left common iliac artery (CIA) following successful wire crossing. C) Catheter-directed thrombolysis of left CIA. D) Left CIA opacification following overnight infusion of tPA. E) Kissing balloon inflation of bilateral CIA. F) Successful restoration of inflow. 
anticoagulation. In one prospective series, 50 consecutive patients on chronic anticoagulation underwent percutaneous coronary interventions via radial access without reversing anticoagulation (mean INR of $2.2 \pm 0.6$ ). There were no complications during the hospital stay for the entire series. There was only one minor bleed, eight days after the procedure, unrelated to the access site..$^{17}$

\section{Additional benefits}

There have been numerous studies from the coronary literature demonstrating cost savings using a radial approach compared to other access sites, but this benefit can be extended to peripheral interventions. The cost reduction derives from the use of fewer resources and shorter hospital stays, partly as a result of decreased bleeding complications that obviate the need for additional, expensive tests (ie, computed tomography scans, ultrasounds, phlebotomy). One recent analysis comparing the TRA to the TFA in cardiac catheterizations noted that the total procedural cost, including access devices, contrast material volume, catheters, closure devices, and recovery, was significantly reduced using the former approach. ${ }^{18}$ In terms of length of stay, a metanalysis showed that the use of the TRA for various patient populations, including elective and emergent coronary interventions, resulted in a significant reduction in the hospital stay by 0.4 days $(P=0.0001) .{ }^{19}$ In our own series of aorto-iliac interventions, the time to discharge was significantly shorter for the TRA group compared to the TFA group (14.4 hr vs $20.9 \mathrm{hr}, P=0.003)$.

\section{Future developments}

The TRA to infrainguinal interventions is still limited by the lack of adequate equipment. As the endovascular field advances, an emphasis must be placed on upgrading the currently available tools. Long marker wires to precisely measure the distance from the entry point to the iliac system are needed, and a larger variety of sheath and wire lengths should be made available. Balloon, stents, and atherectomy devices that can reach the popliteal and proximal tibial vessels, at the very least, should be developed. Rescue catheters have to be long enough to extend into the dorsal and plantar arches. Furthermore, there should be an emphasis on downsizing the caliber of these devices, in order to allow all interventions to be performed through 5 Fr or 6 Fr sheaths. As a final point, training programs that allow for proficiency in performing transradial interventions need to be expanded.

\section{Conclusion}

In the final analysis, the transradial approach to peripheral vascular interventions offers numerous advantages, beyond the well-established lower risk of post-procedural bleeding. We strongly support the use of the radial artery as the access site for interventions to the aorto-iliac vascular bed. More advanced support catheters, larger balloons, and improved stent selection are needed before this field moves on into the superficial femoral artery interventions.

\section{Disclosures}

The authors report no conflicts of interest in this work.

\section{References}

1. Agostoni P, Biondi-Zoccai GG, de Benedictis ML, et al. Radial versus femoral approach for percutaneous coronary diagnostic and interventional procedures; Systematic overview and meta-analysis of randomized trials. J Am Coll Cardiol. 2004;44:349-356.

2. Cox N, Resnic FS, Popma JJ, Simon DI, Eisenhauer AC, Rogers C. Comparison of the risk of vascular complications associated with femoral and radial access coronary catheterization procedures in obese versus nonobese patients. Am J Cardiol. 2004;94:1174-1177.

3. Rao SV, Ou FS, Wang TY, et al. Trends in the prevalence and outcomes of radial and femoral approaches to percutaneous coronary intervention: a report from the National Cardiovascular Data Registry. JACC Cardiovasc Interv. 2008;1:379-386.

4. Staniloae CS, Kurian DC, Coppola JT. Transradial bilateral iliac stenting. J Invasive Cardiol. 2006;18:E256-E257.

5. Staniloae CS, Korabathina R, Yu J, Kurian D, Coppola J. Safety and efficacy of transradial aortoiliac interventions. Catheter Cardiovasc Interv. 2010;75:659-662.

6. Elgharib NZ, Shah UH, Coppola JT. Transradial cardiac catheterization and percutaneous coronary intervention: a review. Coron Artery Dis. 2009;20:487-493.

7. Valsecchi O, Vassileva A, Musumeci G, et al. Failure of transradial approach during coronary interventions: anatomic considerations. Catheter Cardiovasc Interv. 2006;67:870-878.

8. Nie B, Zhou YJ, Li GZ, Shi DM, Wang JL. Clinical study of arterial anatomic variations for transradial coronary procedure in Chinese population. Chin Med J (Engl). 2009;122:2097-2102.

9. Oshima A, Takeshita S, Kozuma K, et al. Intravascular ultrasound analysis of the radial artery for coronary artery bypass grafting. Ann Thorac Surg. 2005;79:99-103.

10. Staniloae CS, Mody KP, Sanghvi K, et al. Histopathologic changes of the radial artery wall secondary to transradial catheterization. Vasc Health Risk Manag. 2009;5:527-532.

11. Trani C, Burzotta F, Tommasino A, Giammarinaro M. Transradial approach to treat superficial femoral artery in-stent restenosis. Catheter Cardiovasc Interv. 2009;74:494-498.

12. Byrne J, Spence MS, Fretz E, et al. Body mass index, periprocedural bleeding, and outcome following percutaneous coronary intervention (from the British Columbia Cardiac Registry). Am J Cardiol. 2009;103:507-511.

13. Achenbach S, Ropers D, Kallert L, et al. Transradial versus transfemoral approach for coronary angiography and intervention in patients above 75 years of age. Catheter Cardiovasc Interv. 2008;72:629-635.

14. Kawashima O, Endoh N, Terashima M, et al. Effectiveness of right or left radial approach for coronary angiography. Catheter Cardiovasc Interv. 2004;61:333-337. 
15. Flachskampf FA, Wolf T, Daniel WG, Ludwig J. Transradial stenting of the iliac artery: a case report. Catheter Cardiovasc Interv. 2005;65: 193-195.

16. Brewster DC. Clinical and anatomical considerations for surgery in aortoiliac disease and results of surgical treatment. Circulation. 1991;83:I42-I52.

17. Helft G, Dambrin G, Zaman A, et al. Percutaneous Coronary Intervention in Anticoagulated Patients via Radial Artery Access. Catheterization and Cardiovascular Interventions. 2009;73:44-47.
18. Roussanov O, Wilson SJ, Henley K, et al. Cost-effectiveness of the radial versus femoral artery approach to diagnostic cardiac catheterization. J Invasive Cardiol. 2007;19:349-353.

19. Jolly SS, Amlani S, Hamon M, Yusuf S, Mehta SR. Radial versus femoral access for coronary angiography or intervention and the impact on major bleeding and ischemic events: a systematic review and metaanalysis of randomized trials. Am Heart J. 2009;157:132-140.

\section{Publish your work in this journal}

Vascular Health and Risk Management is an international, peerreviewed journal of therapeutics and risk management, focusing on concise rapid reporting of clinical studies on the processes involved in the maintenance of vascular health; the monitoring, prevention and treatment of vascular disease and its sequelae; and the involvement of metabolic disorders, particularly diabetes. This journal is indexed on PubMed Central and MedLine. The manuscript management system is completely online and includes a very quick and fair peer-review system, which is all easy to use. Visit http://www.dovepress.com/ testimonials.php to read real quotes from published authors.

Submit your manuscript here: http://www.dovepress.com/vascular-health-and-risk-management-journal 